\title{
QUELQUES ARMES ESPAGNOLES AU MUSÉE D'ART ET D'HISTOIRE DE GENÈVE \\ PAR \\ CLEMENT BOSSON
}

LA collection des armes et armures du Musée d'Art et d'Histoire de Genève provient de l'ancien arsenal de la République. Cette collection est donc composée surtout de pièces qui avaient été utilisées par les soldats des corps de troupe de Genève et des officiers de la République et par les dépouilles des adversaires de la Cité. Les armuriers allemands -et particulièrement italiens- étaient les fournisseurs de l'Etat par l'intermédiaire de marchands établis en ville. C'est la raison pour laquelle les armes construites en Espagne sont peu nombreuses dans ce musée.

Arquebuse À silex (anc. inv, nr. 2226)

Son origine est indiscutable, avec sa crosse catalane ${ }^{1}$ et sa platine méditerranéenne si caractéristique, appelée aussi platine espagnole ou platine à la miquelet. La description française en étant assez rare, nous pensons bien faire en la présentant ici:

«La plaque de batterie, cannelée de stries sur la face battue par la pierre, fait corps avec le couvre-bassinet. Le ressort du chien est à l'extérieur, sa branche longue presse l'arrière du pied du chien de bas en haut et le soulève, le forçant à s'abattre vers la batterie. Le corps du chien, de forme simple et trapue, toujours droit, jamais en $S$, supporte deux fortes mâchoires, traversées par une vis de serrage à anneau. Le pied du chien porte vers l'avant un prolongement en arc de cercle qui s'amincit. En tirant un peu le chien en arrière, cet arc de cercle, qui empêchait la sortie du cran de sûreté, prend appui sur ce cran qui traverse la plaque de platine. Ce verrou est à encoche, de sorte que le chien ne peut être dégagé qu'en continuant le mouvement décrit plus haut; il vient ainsi prendre appui sur le verrou d'armé. En position de sûreté, le chien est suffisamment écarté pour qu'il soit facile de charger le bassinet et de le fermer.

L'action de la détente, sur la tige de gâchette, entraîne la première partie du ressort de gâchette sur lequel est fixé le cran de sûreté qui traverse la plaque de platine. En continuant son action, la tige de gâchette écarte davantage la partie du ressort auquel est soudé le verrou d'armé.

${ }^{1}$ W. Keith Neal: Spanish Guns \& Pistols, pl. 44 à 47. 
Celui-ci est taillé en biseau à son extrémité afin de pouvoir glisser facilement sous le pied du chien et s'effacer à l'intérieur de la plaque de platine. Le chien, forcé par le grand ressort, s'abat alors contre la batterie, allumant l'amorce. La très grande robustesse de ce type de platine explique son emploi général dans la plupart des pays méditerranéens.

Nos connaissances sont minces sur son origine; nous les devons à un armurier de Madrid, Isidro Soler, arquebusier du roi Charles IV (17481819), dès 1792. Il publie à Madrid, en 1795, le Compendio Histórico de los Arcabuceros de Madrid desde su Origen basta la Epoca presente. En voici un passage: '... C'est à Simon Marcuarte, qui fut l'armurier de Philippe II (1527-1598) que l'on doit l'invention des platines à silex que nous nommons espagnoles. Jusqu'alors on ne connaissait que les platines à rouet.'

Le nom de Marcuarte nous est connu par le traité de l'armurier du roi Philippe IV (1605-1655) Martínez Alonso de Espinar, qui publie en 1644, à Madrid, Arte de la Ballestría y Monterío, d'où nos tirons ce qui nous intéresse: '... Les plus fameux maîtres qu'il y eut en Espagne vivaient à Madrid, ils se nommaient maître Simón, le plus âgé et maître Pedro, son frère... Ils furent amenés d'Allemagne en Espagne par l'empereur Charles Quint... et travaillèrent pour le roi Philippc II ct Philippe III... Maître Simón avait quatre fils travaillant tous dans l'armurerie' 2 .

Si ces indications sont exactes, les deux frères seraient venus en Espagne, au plus tard vers 1550 et auraient travaillé pour la cour (Philippe III ayant succédé à son père en 1598) jusque vers 1600 au moins. Il est donc fort possible que, né vers 1520-1525, leur réputation les amena au-delà des Pyrénées vers le milieu du siècle.

Maître Simón l'Ancien (env. 1525-env. 1600 selon les renseignements ci-dessus) avait donc quatre fils dont le troisième se prénommait aussi Simón. Si l'on admet sa naissance vers 1560 , c'est au début du XVII ${ }^{\mathrm{C}}$ siècle qu'il avait acquis les connaissances et l'habileté créatrice pour l'invention de la platine espagnole qui lui est attribuée par Isidro Soler.»

La plaque de batterie de notre arquebuse est carrée, le cran de mire, à la française, est en argent, soudé sur une plaquette rectangulaire encastrée dans le canon, la hausse est à cheval sur un motif ciselé. La dimension de l'arme explique la présence du crochet de suspension:

$$
\begin{aligned}
& \text { Longuer totale ................ } 86 \mathrm{~cm} \text {. } \\
& \text { Longuer du canon ........... } 54 \mathrm{~cm} \text {. } \\
& \text { Calibre ...................... } 15 \mathrm{~mm} \text {. }
\end{aligned}
$$

Cette arquebuse ne porte pas de poinçon.

2 J.-F. Hayward: Les Armes à feu anciennes, vol. I, p. 124. 


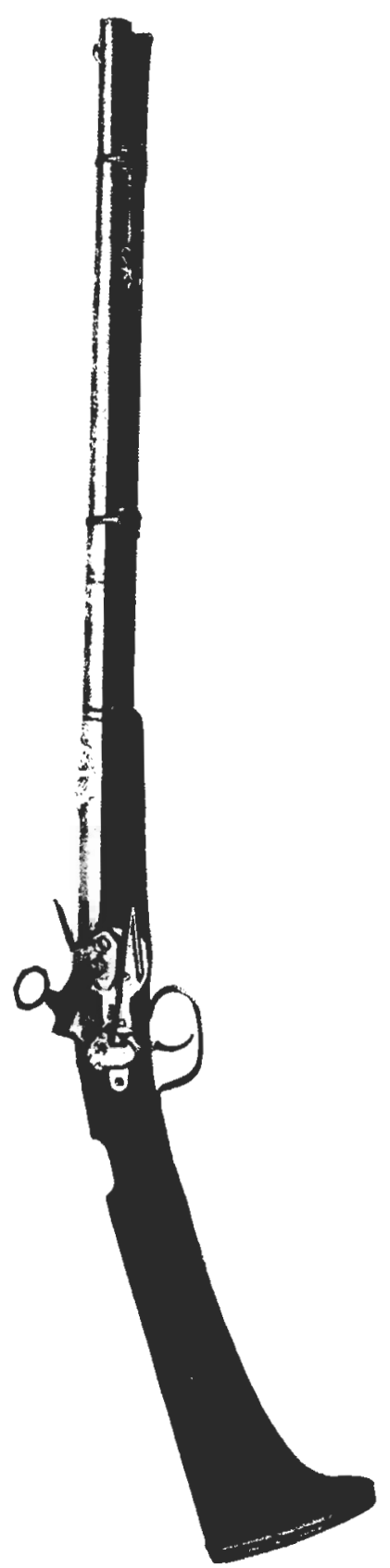

Fig. 1.-Arquebuse à silex (anc. inv. nr. 2226). (Phot. Yves Digitalizado por InterClassica http://interclassica.um.es

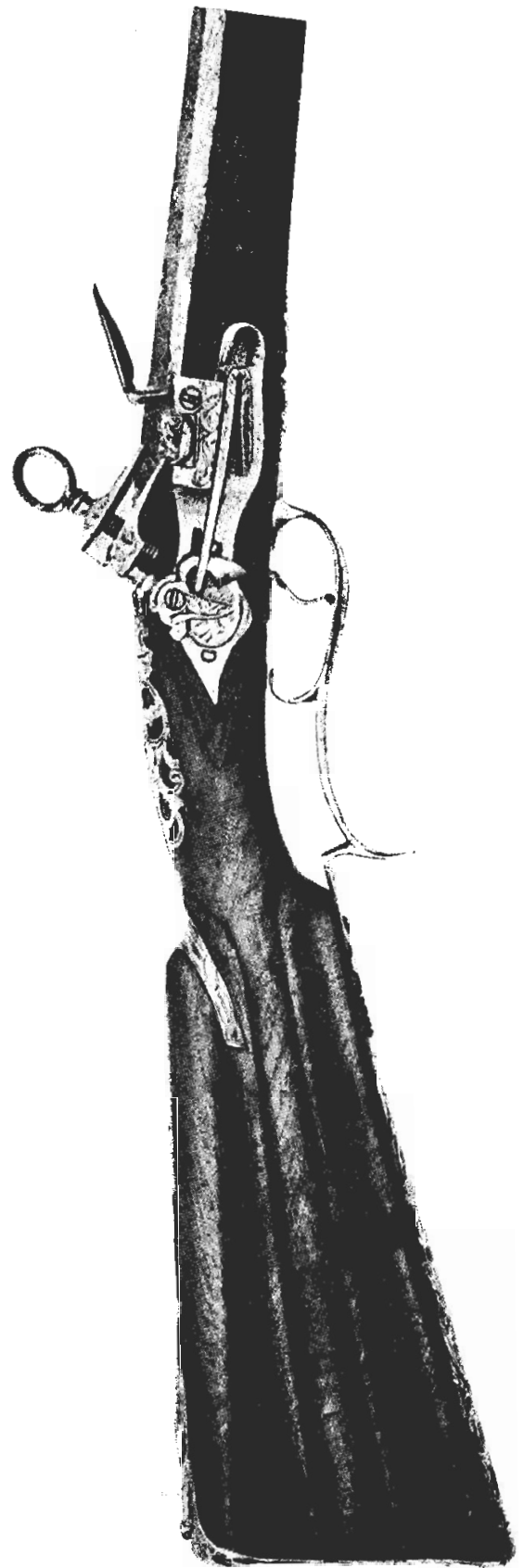

FIG 2.-Fusil à silex avec le poinçon de Sala. (Phot. Yves Siza.)

Consejo Superior de Investigaciones Científicas http://gladius.revistas.csic.es 
Fusil ̀̀ SILEX

Sur le canon est insculpé le poinçon SALA sous une couronne. Cette marque est représentée dans la liste de Monsieut W. Keith Neal sous le nr. $155^{3}$, avec la date: env. 1770 , mais sans précision quant au lieu de travail de l'armurier. Le médaillon en argent de la plaque de pouce se retrouve sur la garniture de crosse en laiton, ainsi que sur la large contreplatine.

La table du couvre-bassinet est de la forme «à la française», mais la surface de contact avec le silex est striée:

$\begin{array}{lr}\text { Longueur totale } \ldots \ldots \ldots \ldots \ldots & 147,5 \mathrm{~cm} . \\ \text { Canon } \ldots \ldots \ldots \ldots \ldots \ldots \ldots \ldots \ldots & 107 \mathrm{~cm} . \\ \text { Calibre } \ldots \ldots \ldots \ldots \ldots \ldots \ldots \ldots & 1,5 \mathrm{~cm} .\end{array}$

Paire de PISTOLETS À SILex

Les canons sont signés AÑO YRUSTA 1803.

La platine porte de nom de ARANA alors que la garniture a été réaljsée par ZIARRA.

Ces pistolets sont munis de crochets de ceinture, il n'y a pas d'appareil de visée. A l'extrémité arrière des plaques de platine existe encore la rosace qui servait à retenir une protection de la main, en forme de petit coussin ovale, et qui allait de la fixation jusque contre le chien ${ }^{4}$.

Le platineur ARANa est cité par Stoeckel, avec les dates 1730-1770. Ces dates correspondent à la construction des montures des platines, alors que les canons originaux ont été remplacés environ 50 ans après la mise en service de ces armes par le canonnier Yrusta qui travaillait à Eibar,

Dimensions:

$\begin{array}{lr}\text { Longueur totale } \ldots \ldots \ldots \ldots \ldots . . & 200 \mathrm{~mm} . \\ \text { Longueur du canon ........... } & 107 \mathrm{~mm} . \\ \text { Calibre ..................... } & 16 \mathrm{~mm} .\end{array}$

3 W. KeIth NenL: Spanish Guns \& Pistols, p. 96.

${ }^{4}$ Ce coussir de protection est visible sur le pistolet de Ripoll de la collection James D. Lavin et reproduit dans son livre: $A$ History of Spanisb Firearms, London 1965, p. 252. 


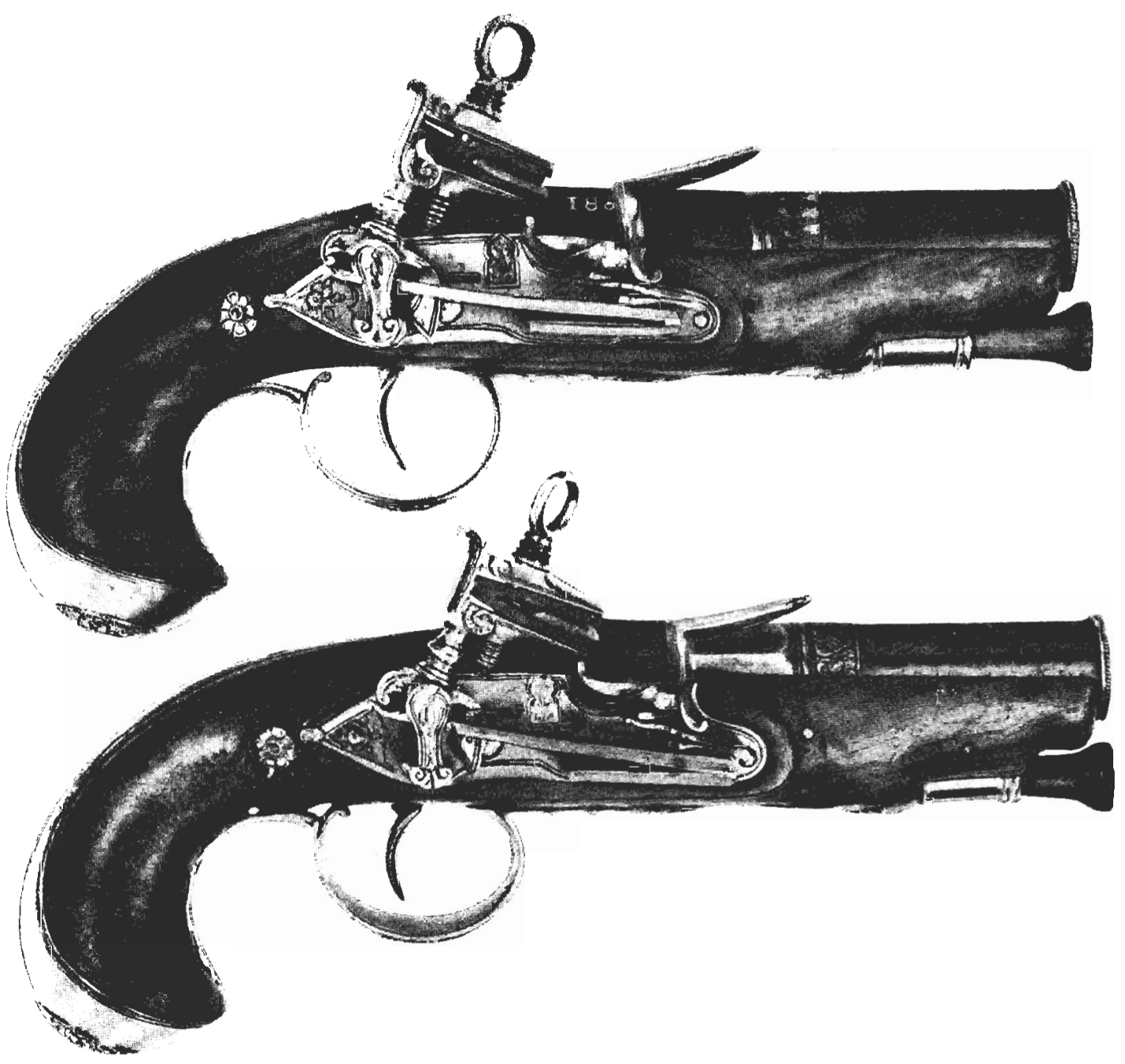

Fig. 3.-Paire de pistolets à silex, signés: año Yrusıa 1803. (Phot. Yves Siza.) 


\section{Paire de Pistolets de Ripoll}

Le canon de nos pistolets est à pans coupés sur la moitié de sa longuer et ensuite rond avec une arête de visée et un renfort à la bouche. La table du couvre-bassinet, datée 1708 est striée. Le revêtement du fût est en argent, ce qui est très rare. Les canons sont signés COMA, nom qui

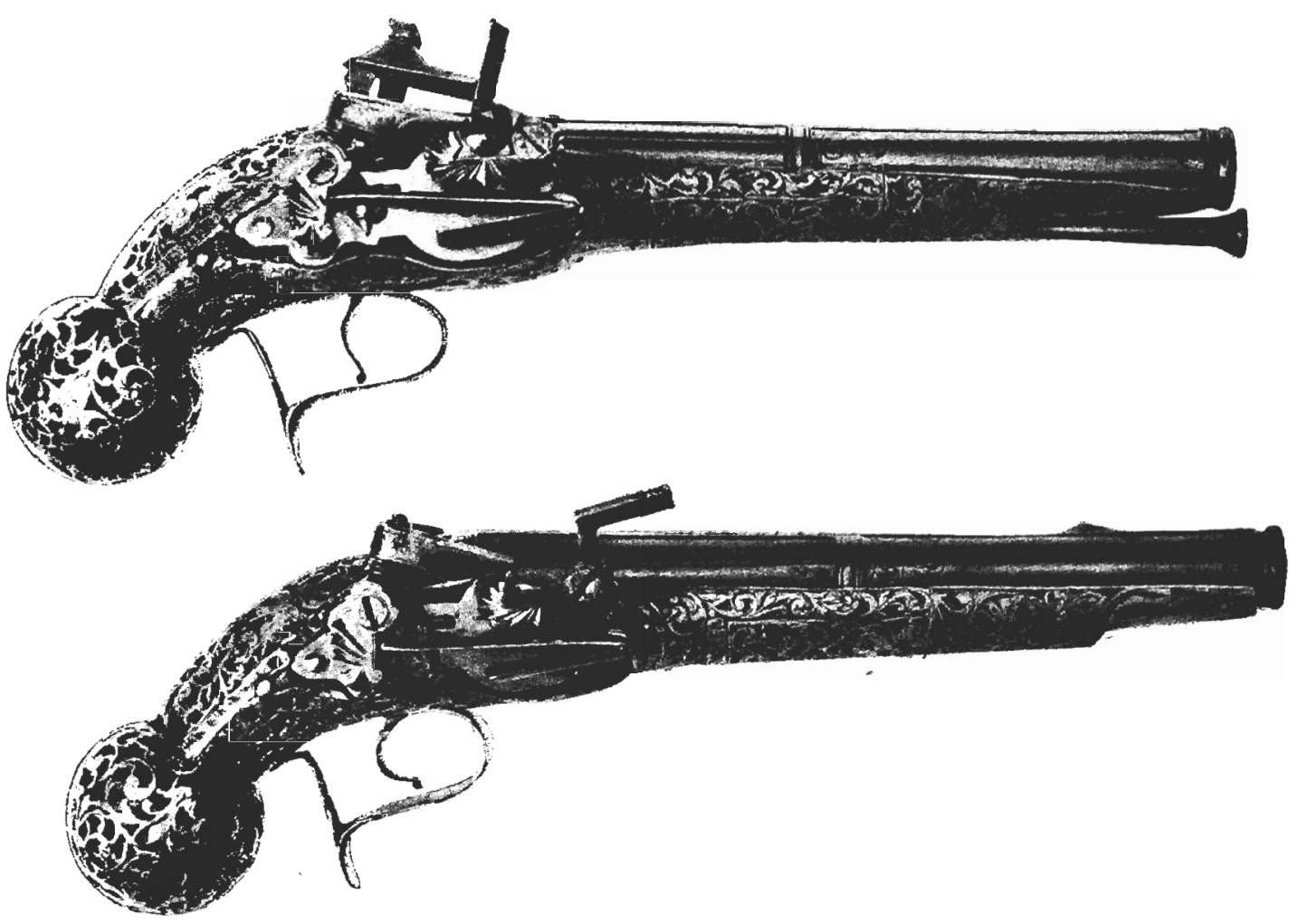

FIG. 4.--Paire de pistolets de Ripoll. (Phot. Yves Siza.)

se retrouve sur un pistolet et sur une paire de la collection Pauilhac. La platine porte le nom de Bonani - dont nous ne savons rien- pas plus que de Pera Carbunel, auteur de la monture. 
En voici les dimensions:

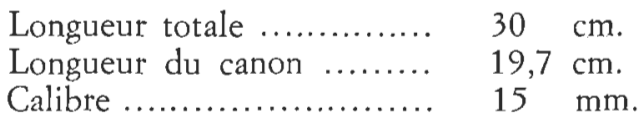

Ripoll est une petite ville d'Espagne située sur la route de Toulouse à Barcelonne, à $65 \mathrm{~km}$. de la frontière française. On y visite le fameux monastère de Santa María, fondé en 589 par Recarede, détruit à plusieurs reprises et restauré depuis 1883 .

Rien n'y subsiste de son industrie armurière qui, dès la fin du XvI ${ }^{\mathrm{e}}$ siècle, en fit une cité aussi importante que Gardonne.

En effet, Ripoll eut à souffrir de destructions entre 1809 et 1813 , au cours de ses luttes contre les français. Cependant, la fabrication continue à un rythme ralenti jusqu'à la guerre civile de 1833 . En 1835 et en 1839 , Ripoll subit l'assaut des troupes de Don Carlos (1788-1855) prétendant au trône à la mort de son frère, le roi Ferdinand VII. La ville fut alors complètement détruite par le feu et par les mines et ce fut la fin des ateliers de fabrication d'armes

Ripoll a produit des arquebuses et des pistolets avec platines à rouet, à silex, à percussion. Il est possible de connaître les premières arquebuses à rouet de RipolL en examinant l'arme M-109 du Musée de l'Armée de Paris ${ }^{5}$; sa crosse est très arquée, entièrement sculptée, ainsi que le fût.

Les pistolets à rouet de ce centre présentent des caractéristiques très distinctes. Une crosse assez mince, courte, se terminant par un pommeau qui s'écarte en deux petits prolongements, dans le plan de l'arme. Le canon n'est pas dans l'axe de la crosse mais est fixé sur la gauche de celle-ci afin de laisser sa place à une lourde platine à l'extérieur du bois, rétablissant ainsi l'équilibre des poids.

Nous connaissons trois armes de ce modèle, l'une à l'armerie de Madrid avec une longeur totale de $96,3 \mathrm{~cm}$., l'autre à Londres au Victoria \& Albert Museum (inv. M-487-1927) longue de $56 \mathrm{~cm}$. et la troisième, datée 1614, au musée Correr à Venise. Leur dimension obligeait à soutenir l'arme de la main ou du bras gauche.

Cependant, les armuriers de Ripoll ne fabriquaient pas que ce type de crosse. Le Scottish Royal Museum d'Edimbourg conserve un pistolet avec un canon de $39 \mathrm{~cm}$. de long. La crosse est d'un incurvé arrondi avec un pommeau ovoïde, le tout un peu de la forme du pistolet saxon, mais moins lourd, plus élancé; la prise était normale avec le pommeau à l'extérieur de la main.

"Reprod.: Armes et armures anciennes..., vol. II, 1927, pI. XLIII. 
Dans la collection Georges Pauilhac, la crosse d'un pistolet à rouet est déjà très proche de ce que l'on peut qualifier de «classique» de Ripoll. Le pommeau est ovale mais trouve sa place dans le creux de la main, le médius maintenant l'arme par le doigtier en crochet à l'arrière du pontet de sous-garde.

Le type classique de Ripoll

Le pistolet de Ripoll — comme ceux de Genève- correspond à l'appellation classique du nom et a été créé en même temps que la platine méditerranéenne. Donc, au début du Xvi $I^{*}$ siècle, les armutiers de Ripoll «sortent» leur modèle à crosse très courte $(10$ à $13 \mathrm{~cm}$.), à pommeau rond plus facile à loger au creux de la main que la forme ovoïde. La décoration des fûts est absolument caractéristique: résille de fer, de cuivre, parfois d'argent, revêtement plein, gravé ou ciselé, ou bois sculpté.

Presque toujours, les pistolets de Ripoll sont complétés par un long crochet de baudrier, le même que celui du douanier dessiné par Goya dans son carton pour tapisserie: El Resgucrdo de Tabacos.

\section{Pistolets de Ripoll à percussion}

La collection Pauilhac, dont l'extraordinaire richesse est une mine de renseignements dans tant de domaines, nous montre le dernier stade de la fabrication de Ripoll, arrêtée, comme nous l'avons vu, en 1835. Il s'agit d'un pistolet de poche, à percussion, qui a gardé toutes les caractéristiques que nous venons d'esquisser. Le revêtement métallique du fût est en cuivte ciselé.

\section{EPÉE EN!. 1650}

La lame est signée enrique coel en alamania. Wendelin Boeheim consacre un court article à cet armurier, dans son ouvrage: Meister der Waffenscbmiedekunst ${ }^{6}$, toutefois sans aucune précision.

Nous ne connaissons ce forgeur de lame que par sa signature. Celle-ci est tout d'abord typiquement allemande: HEINRICH KOEL 1617 MEFECIT SOLINGEN 1617; elle se lit sur la lame d'une épée du château de Windsor (36A). Selon les indications de Guy-Francis Laking, auteur des notices

'Wendelin Boeneim: Meister der Waffenscbmiedekunst..., Berlin 1897, p. 36. 


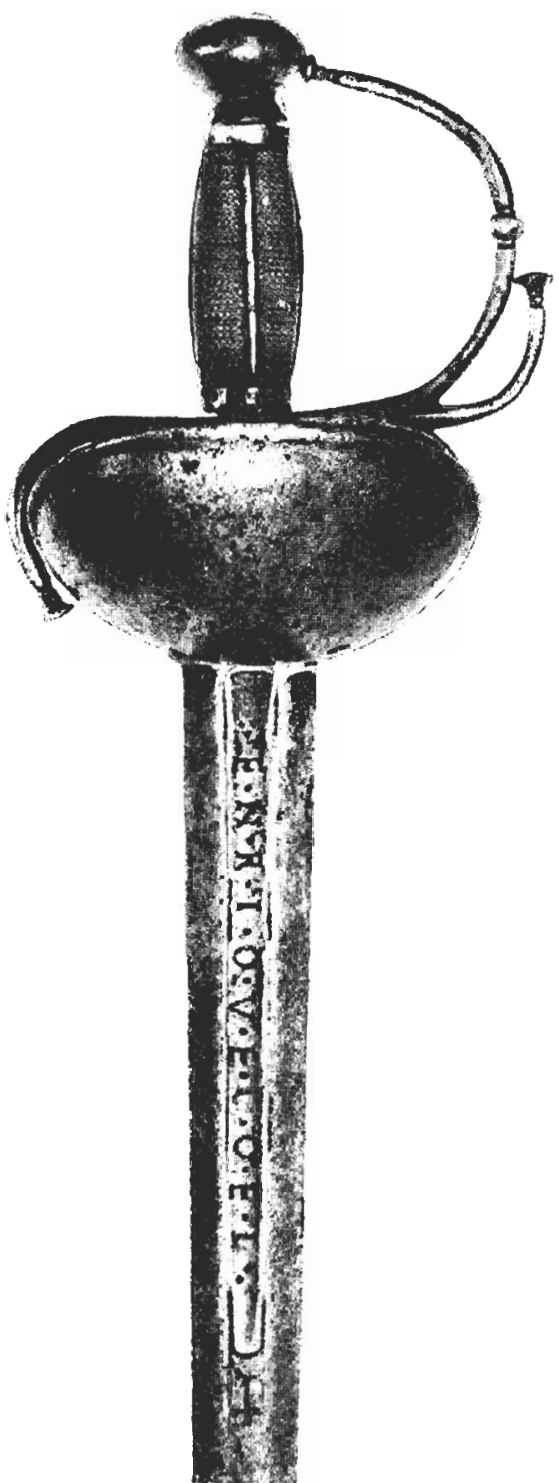

FIG. 5.--Epée espagnole avec la signature: «Enrique Coel en Alamania». (Phot. Yves Siza.)

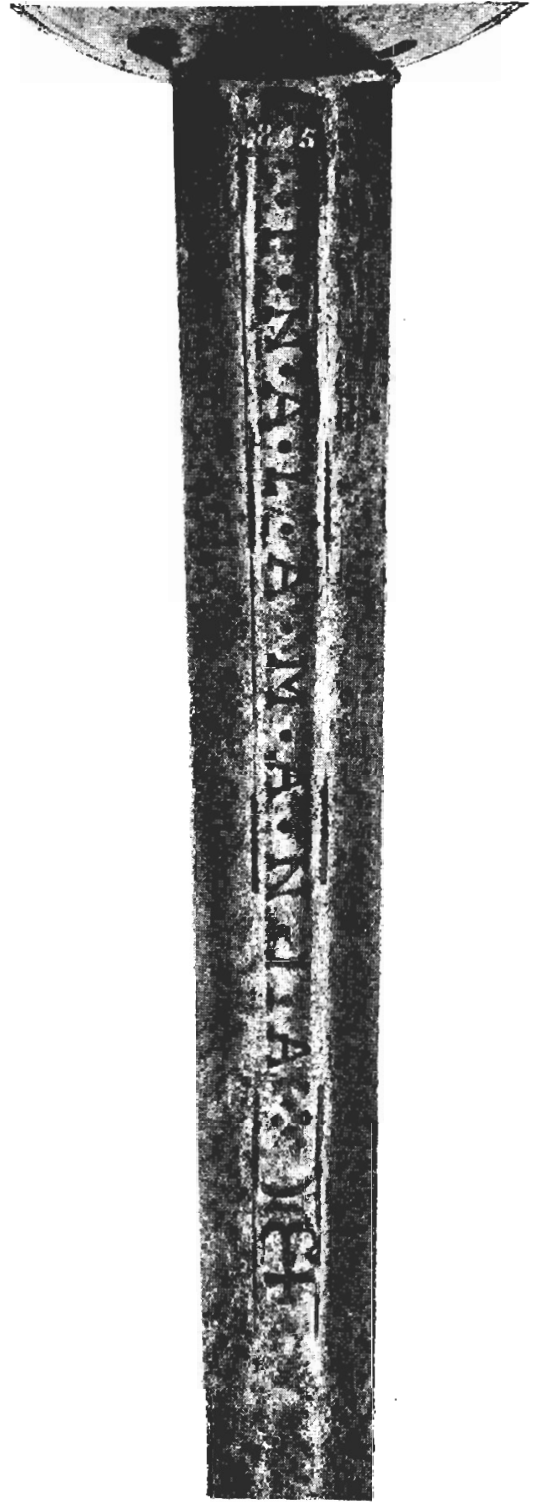

FIG. 6.-Lame de l'épée fig. 5 avec l'inscription. (Phot. Yves Siza.) 
sur les armes de Windsor ${ }^{7}$ la monture serait antérieure à la lame. Celle-ci, si l'on se réfère à l'hispanisme qui va suivre de sa signature, correspond à l'une de ses premières oeuvres.

Dans cette même collection, existe une magnifique paire d'armes: l'épéc est à garde en coquille d'acier plein délicatement décoré de motifs guerriers. La main gauche est réalisée «en suite». Cet ensemble aurait appartenu à Philippe IV d'Espagne (1621-1665) et proviendrait de l'armerie de Don Manuel de Godoy, Prince de la Paix. La lame porte la signature encore bien allemande: HEINRICH COEL ME FECIT SOLINGEN ${ }^{8}$.

Encore une inscription allemande sur la lame de l'épée à garde coquille avec rebord en brise-lame de la collection Spitzer (No. 204) HEINRICH COLL. Ce sont les seules signatures entièrement germaniques. A Vienne, la lame de l'épée à coquille A 1463, est signée ENRICO GOEL SOLINGEN.

L'accentuation espagnole de la signature est marquée par l'inscription ENRIQUE COLL ESPADERO EN ALEMANIA .

En revenant au château de Windsor, nous trouvons encore une épée dont la monture, de la fin du XiII ${ }^{\mathrm{e}}$ siècle, a hérité d'une lame avec l'inscription ENRITE COL ESPADERO DEL REY EM ALLEMANHA (183A).

Une gravure semblable se lit sur une épée du Musée de l'Armée à Paris (J. 182) ${ }^{10}$ ENRIQue COL ESPADERo DEL REY EIN ALlEMANAH.

Ce sont les seules références, à notre connaissance, au titre de notre armurier «fournisseur du roi». Mais les lames sont-elles bien de Heinrich Coel? S'agit-il d'une signature «imitée» comme il y en eut tant?

La répétition des noms Solingen et Alamania semble prouver que le forgeur de lames n'a jamais quitté l'Allemagne, tout en étant fournisseur attitré du roi d'Espagne, pour autant que la paire d'armes ait bien appartenu à Philippe IV.

Les deux auteurs qui se sont occupé de cet armurier, Wendelin Boeheim et Albert Weyersberg, ne disent rien de précis à son sujet.

3 The Armoury of Windsor Castle, London 1904.

${ }^{8} 67$ et $68 \mathrm{a}$.

- Metropolttan Museum of Art of New York: Calalogue exposition de Hagerstown, Mayland 1955, no. 63.

${ }^{10}$ L. RonerT: Catalogue des collections composant le Musée d'Artillerie 1889, Paris 1891, t. III, p. 67. 


\section{B I B L I O G R A P I E}

Charles Butrin: Armes à feu el armes blanches. No. 17, 1914.

Le complément de l'étude de Charles Buttin n'a jamais été publié. Le responsable de la revue, Monsieur F. Thevin écrivait, le 27 août 1914: «... Je dois suspendre la publication, comme tous mes confrères d'ailleurs, car l'imprimerie est arrêtée faute de personnel et de papier... tous mes collaborateurs officiers sont à la frontic̀re...»

Il semble qu’un spécialiste a accepté de compléter les notes de Charies Buttin sur Ripoll par des recherches dains les collections; ce travail serait très apprécié.

G. Macoir: Pistolets catalans de Ripoll, in «Bulletin des Musées Royaux du Cinquantenaire», avril 1914.

Thomas T. Hoopes: Ripollscbe Radscblosspistolen, in Zeitscbrift für Historiscbe Waffen- und Kostumkunde. N. F., Band IV, 1932-1934, Heft 10, p. 227.

Claude Bair: Pistols of the World. 1968 , pl. 123 et suiv.

En terminant cette étude, qu'il me soit permis de remercier très sincèrement Monsieur Eugen Heer, assistant-conservateur dı Musée d'Art et d'Histoire de Genève, pour l'aide précieuse qu'il a bien voulu m'apporter. 